\title{
On the Solvability of the Boundary-Value Problem for the First Order Operator-Differential Equations with Multiple Characteristics
}

\author{
Mohamed A. Labeeb ${ }^{1}$, Ahmed L. Elbably², Abdel Baset I. Ahmed1 \\ ${ }^{1}$ Egyptian Russian University, Badr City, Egypt \\ ${ }^{2}$ Helwan University, Helwan, Egypt \\ Email:m.labeeb85@yahoo.com,Ahmed_Elbably2004@yahoo.com,abdel2007@yandex.ru
}

How to cite this paper: Labeeb, M.A., Elbably, A.L. and Ahmed, A.B.I. (2017) On the Solvability of the Boundary-Value Problem for the First Order Operator-Differential Equations with Multiple Characteristics. Open Access Library Journal, 4: e4155.

https://doi.org/10.4236/oalib.1104155

Received: November 15, 2017

Accepted: December 16, 2017

Published: December 19, 2017

Copyright $\odot 2017$ by authors and Open Access Library Inc.

This work is licensed under the Creative

Commons Attribution International License (CC BY 4.0).

http://creativecommons.org/licenses/by/4.0/

\begin{abstract}
In this paper, a class of operator-differential equation of the first order with multiple characteristics is considered, for which the initial boundary value problem on the semi-axis is well-posed and uniquely solvable in the Sobolev space.
\end{abstract}

\section{Subject Areas \\ Ordinary Differential Equation}

\section{Keywords}

Operator-Differential Equation, Boundary-Value Problem, Hilbert Space, Regular Solvability

Suppose that $H$ is a separable Hilbert space with a scalar product $(x, y)$, $x, y \in H, A$ is a self-adjoint non negative definite operator on $H$ ( $A=A^{*} \geq c E, c>0, E$ is the identity operator). By $H_{\gamma} \quad(\gamma \geq 0)$, we denote the scale of Hilbert spaces generated by the operator $A$, i.e. $H_{\gamma}=\operatorname{Dom}\left(A^{\gamma}\right)$,

$(x, y)_{\gamma}=\left(A^{\gamma} x, A^{\gamma} y\right), \quad x, y \in \operatorname{Dom}\left(A^{\gamma}\right)$. For $\gamma=0$ we consider that $H_{0}=H$, $(x, y)_{0}=(x, y), \quad x, y \in H$.

By $L_{2}([e, b] ; H)(-\infty \leq e<b \leq+\infty)$, we denote the space of measurable functions (see [1]) with values in $H$ and the norm

$$
\|f\|_{L_{2}([a, b] ; H)}=\left(\int_{e}^{b}\|f(t)\|^{2} \mathrm{~d} t\right)^{1 / 2},
$$


$W_{2}^{1}([e, b] ; H)$ is the space of all functions with values in $H$ such that $\frac{\mathrm{d} u(t)}{\mathrm{d} t}$, $A u(t) \in L_{2}([e, b] ; H)$ with the norm

$$
\|u\|_{W_{2}^{1}([e, b] ; H)}=\left(\left\|\frac{\mathrm{d} u}{\mathrm{~d} t}\right\|_{L_{2}([a, b] ; H)}^{2}+\|A u\|_{L_{2}([a, b] ; H)}^{2}\right)^{1 / 2} .
$$

For more details about the space $W_{2}^{1}([e, b] ; H)$, see $[[2]$, Ch.1]. We assume that

$$
\begin{aligned}
& \text { For } e=-\infty, b=+\infty \\
& L_{2}((-\infty,+\infty) ; H) \equiv L_{2}(R ; H), W_{2}^{1}((-\infty,+\infty) ; H) \equiv W_{2}^{1}(R ; H), R=(-\infty,+\infty), \\
& \text { For } e=0, b=+\infty \\
& \quad L_{2}([0,+\infty) ; H) \equiv L_{2}\left(R_{+} ; H\right), W_{2}^{1}([0,+\infty) ; H) \equiv W_{2}^{1}\left(R_{+} ; H\right), R_{+}=[0,+\infty) .
\end{aligned}
$$

For the whole article, all the derivatives are understood in the sense of distributions.

Consider the following problem on the semiaxis $R_{+}$:

$$
\begin{gathered}
\frac{\mathrm{d} u(t)}{\mathrm{d} t}+A u(t)=f(t), t \in R_{+}, \\
u(0)=0,
\end{gathered}
$$

where $A=A^{*} \geq c E, c>0, f(t) \in L_{2}\left(R_{+} ; H\right), u(t) \in W_{2}^{1}\left(R_{+} ; H\right)$.

Definition 1. If the vector function $u(t) \in W_{2}^{1}\left(R_{+} ; H\right)$ satisfies Equation (1) nearly everywhere in $R_{+}$, then it is called a regular solution of Equation (1).

Definition 2. For any $f(t) \in L_{2}\left(R_{+} ; H\right)$ there exists a regular solution $u(t)$ of Equation (1) satisfying the boundary Conditions (2) in the sense of relation

$$
\lim _{t \rightarrow 0}\left\|A^{1 / 2} u(t)\right\|=0
$$

and the following inequality holds:

$$
\|u\|_{W_{2}^{1}([e, b] ; H)} \leq \text { const }\|f\|_{L_{2}\left(R_{+} ; H\right)},
$$

Then we say that say that problem (1), (2) is regularly solvable.

In this paper, the regular solvability of the initial boundary value problem (1), (2) is established.

In spite of the fact that, since 50th years of the last century, there are sufficiently many papers and books dedicated to the solvability of parabolic operator-differential equations in Banach spaces, in particular, Hilbert spaces, interest in such equations doesn't abate until recently (see, for example, [3] [4] [5]). However, in the mathematical literature studies on the parabolic operator-differential equations with multiple characteristics are almost not mentioned, although they have a wide application in some problems in mechanics and mathematical physics. Note that Equation (1) is an abstract parabolic equation in Hilbert space that has multiple characteristics.

We let

$$
\stackrel{o}{W_{2}^{1}}\left(R_{+} ; H\right)=\left\{u(t): u(t) \in W_{2}^{1}\left(R_{+} ; H\right), u(0)=0\right\} .
$$


And denote by $Q_{0}$ the operator acting from the space $\stackrel{o}{W}_{2}^{1}\left(R_{+} ; H\right)$ to the space $L_{2}\left(R_{+} ; H\right)$ as follows:

$$
Q_{0} u(t) \equiv \frac{\mathrm{d} u(t)}{\mathrm{d} t}+A u(t), u(t) \in \stackrel{o}{W_{2}^{1}}\left(R_{+} ; H\right) .
$$

Then the following theorem holds

Theorem 1. The operator $Q_{0}$ is an isomorphism between the spaces $W_{2}^{1}\left(R_{+} ; H\right)$ and $L_{2}\left(R_{+} ; H\right)$.

Proof. Obviously, the equation $Q_{0} u(t)=0$, has only the trivial solution $u(t) \in \stackrel{o}{W_{2}^{1}}\left(R_{+} ; H\right)$. Really, the equation $Q_{0} u(t)=0$ has a solution in the space $W_{2}^{1}\left(R_{+} ; H\right)$ of the form $u_{0}(t)=\mathrm{e}^{-t A} \varphi$, where the vectors $\varphi \in H_{1 / 2}$. Taking into account conditions (??), we have $\varphi=0$ and $u_{0}(t)=0$. Therefore, the equation $Q_{0} u(t)=0$ has only the trivial solution in the space $\stackrel{o}{W_{2}^{1}}\left(R_{+} ; H\right)$.

Now we shall show that the equation $Q_{0} u(t)=f(t)$ for any $f(t) \in L_{2}\left(R_{+} ; H\right)$ has a solution in the space $W_{2}^{1}\left(R_{+} ; H\right)$. We extend the function $f(t)$ to zero for $t<0$, then, applying the direct and inverse Fourier transform, it becomes clear that

$$
u_{1}(t)=\frac{1}{2 \pi} \int_{-\infty}^{+\infty}(i \xi E+A)^{-1}\left(\int_{0}^{+\infty} f(s) \mathrm{e}^{-i \xi s} \mathrm{~d} s\right) \mathrm{e}^{i \xi t} \mathrm{~d} \xi, t \in R
$$

Satisfies the equation $Q_{0} u(t)=f(t)$ almost everywhere in $R$. We show that $u_{1}(t) \in W_{2}^{1}(R ; H)$. From Parseval's equality we obtain:

$$
\begin{aligned}
\left\|u_{1}\right\|_{W_{2}^{2}(R ; H)}^{2}= & \left\|\frac{\mathrm{d} u_{1}}{\mathrm{~d} t}\right\|_{L_{2}(R ; H)}^{2}+\left\|A u_{1}\right\|_{L_{2}(R ; H)}^{2} \\
= & \left\|i \xi \tilde{u}_{1}(\xi)\right\|_{L_{2}(R ; H)}^{2}+\left\|A \tilde{u}_{1}(\xi)\right\|_{L_{2}(R ; H)}^{2} \\
= & \left\|i \xi(i \xi E+A)^{-1} \tilde{f}(\xi)\right\|_{L_{2}(R ; H)}^{2}+\left\|A(i \xi E+A)^{-1} \tilde{f}(\xi)\right\|_{L_{2}(R ; H)}^{2} \\
\leq & \sup _{\xi \in R}\left\|i \xi(i \xi E+A)^{-1}\right\|_{H \rightarrow H}^{2}\|\tilde{f}(\xi)\|_{L_{2}(R ; H)}^{2} \\
& +\sup _{\xi \in R}\left\|A(i \xi E+A)^{-1}\right\|_{H \rightarrow H}^{2}\|\tilde{f}(\xi)\|_{L_{2}(R ; H)}^{2},
\end{aligned}
$$

where $\tilde{u}_{1}(\xi)$ and $\tilde{f}(\xi)$ are the Fourier transforms of the functions $u_{1}(t)$ and $f(t)$, respectively. $\sigma(A)$ denotes the spectrum of the operator $A$. From the spectral decomposition of the operator $A$ for $\xi \in R$, we have:

$$
\begin{aligned}
& \left\|i \xi(i \xi E+A)^{-1}\right\|=\sup _{\mu \in \sigma(A)}\left|i \xi(i \xi+\mu)^{-1}\right| \leq \sup _{\mu \in \sigma(A)} \frac{|\xi|}{\left(\xi^{2}+\mu^{2}\right)^{\frac{1}{2}}} \leq 1, \\
& \left\|A(i \xi E+A)^{-1}\right\|=\sup _{\mu \in \sigma(A)}\left|\mu(i \xi+\mu)^{-1}\right| \leq \sup _{\mu \in \sigma(A)}\left(\frac{\mu^{2}}{\xi^{2}+\mu^{2}}\right)^{\frac{1}{2}} \leq 1 .
\end{aligned}
$$

Taking into account (4) and (5) into (3) we obtain: 


$$
\left\|u_{1}\right\|_{W_{2}^{1}([e, b] ; H)}^{2} \leq 2\|\tilde{f}(\xi)\|_{L_{2}(R ; H)}^{2}=2\|f(t)\|_{L_{2}\left(R_{+} ; H\right)}^{2} .
$$

Therefore, $u_{1}(t) \in W_{2}^{1}(R ; H)$. Now we denote the restriction of the function $u_{1}(t)$ to $R_{+}$by $v(t)$. Obviously, $v(t) \in W_{2}^{1}\left(R_{+} ; H\right)$. By the theorem on traces [[2], Ch.1] $v(0) \in H_{1 / 2}$. Then we will find a solution to the equation $Q_{0} u(t)=f(t)$ of the form

$$
u(t)=v(t)+\mathrm{e}^{-t A} \psi,
$$

where the vectors $\psi \in H_{1 / 2}$. For a clear determination of $\psi$ from conditions (2) we obtain that $v(0)+\psi=0$,

We have

$$
\psi=-v(0) \in H_{1 / 2},
$$

Thus,

$$
u(t) \stackrel{o}{W_{2}^{1}}\left(R_{+} ; H\right) .
$$

It is clear that the operator $Q_{0}$ is bounded and acts from the space $\stackrel{o}{W_{2}^{1}}\left(R_{+} ; H\right)$ to the space $L_{2}\left(R_{+} ; H\right)$. Indeed, taking into account the theorem on intermediate derivatives [[2], Ch.1], we have:

$$
\begin{aligned}
\left\|Q_{0} u\right\|_{L_{2}\left(R_{+} ; H\right)} & =\left\|\frac{\mathrm{d} u}{\mathrm{~d} t}+A u\right\|_{L_{2}\left(R_{+} ; H\right)} \leq\left\|\frac{\mathrm{d} u}{\mathrm{~d} t}\right\|_{L_{L_{2}\left(R_{+} ; H\right)}}+\|A u\|_{L_{2}\left(R_{+} ; H\right)} \\
& \leq \mathrm{const}\|u\|_{W_{2}^{1}\left(R_{+} ; H\right)} .
\end{aligned}
$$

Thus, the operator $Q_{0}: \stackrel{o}{W_{2}^{1}}\left(R_{+} ; H\right) \rightarrow L_{2}\left(R_{+} ; H\right)$ is bijective and bounded. Hence, using the Banach inverse operator theorem, the operator $Q_{0}^{-1}: L_{2}\left(R_{+} ; H\right) \rightarrow W_{2}^{1}\left(R_{+} ; H\right)$ is bounded. Therefore, the operator $Q_{0}$ is an isomorphism between the spaces $W_{2}^{1}\left(R_{+} ; H\right)$ and $L_{2}\left(R_{+} ; H\right)$.

The theorem is proved.

Corollary 2. It follows from Theorem 1 that initial-boundary value problem (1), (2) is regularly solvable.

Corollary 3. It follows from Theorem ${ }_{o} 1$ that the norms $\|u\|_{W_{2}^{1}\left(R_{+} ; H\right)}$ and $\left\|Q_{0} u\right\|_{L_{2}\left(R_{+} ; H\right)}$ are equivalent on the space $W_{2}^{1}\left(R_{+} ; H\right)$.

\section{References}

[1] Hille, E. and Fillips, R.S. (1957) Functional Analysis and Semi-Groups. Amer. Math. Soc. Coll. Publ., Vol. 31, Amer. Math. Soc., Providence, 1957; Izdat. Inostran. Lit., Moscow, 1962.

[2] Lions, J.-L. and Magenes, E. Non-Homogeneous Boundary Value Problems and Applications. Dunod, Paris, 1968; Mir, Moscow, 1971; Springer, Berlin, 1972.

[3] Amann, H. (2005) Maximal Regularity and Quasilinear Parabolic Boundary Value Problems, Recent Advances in Elliptic and Parabolic Problems. World Scientific, Hackensack, 1-17.

[4] Sasun Yakubov, U. (1999) An Initial Boundary Value Problem for Parabolic Differential-Operator Equations. Journal of Mathematical Analysis and Applications, 239, 272-290.

[5] Yakubov, S.Ya. (1985) Linear Differential-Operator Equations and Their Applications. Elm, Baku. (In Russian) 